MATEC Web of Conferences 21, 04004 (2015)

DOI: $10.1051 /$ matecconf $/ 20152104004$

(C) Owned by the authors, published by EDP Sciences, 2015

\title{
Laser bending process of preloaded sheet metal
}

\author{
Lang $\mathrm{Xu}$, Weidong $\mathrm{Li}^{\mathrm{a}}$, Min Wan, Xiufeng Wang, and Yaoguo Dong \\ School of Mechanical Engineering and Automation, Beihang University, Beijing 100191, China
}

\begin{abstract}
Laser bending process of preloaded sheet metal was reviewed on theoretical researches, forming experiments, numerical simulations and material performance studies of formed sheets. Considering the pre-bending platforms that used in the forming experiments can only work on few simple pre-bending types and small sized sheet metals, a large and flexible pre-bending platform was developed by authors. Experiments were done on this platform. The results of experiments showed that different prebending types lead to bending sheet metals into different curved shapes and large sized structure components of flight vehicles can be formed using this process. Based on the current research status, further developments and challenges of this process are discussed.
\end{abstract}

\section{Introduction}

Laser bending process of preloaded sheet metal is a relatively new forming process and its forming procedure is as follows (Fig. 1). The sheet metal is bended in the elastic range by a fixture so that elastic pre-stress turns up inside. Then the elastic strain energy focused regions of sheet metal is scanned by laser spot. With the heating effect of laser, temperature of the scanned regions rises and yield limit of the regions drops, therefore plastic deformation occurs [1].

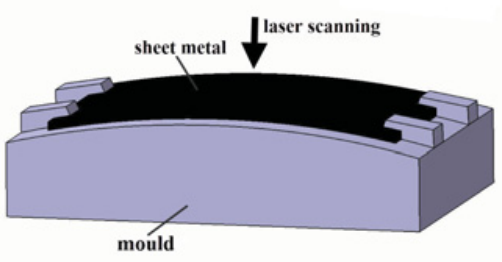

(a)

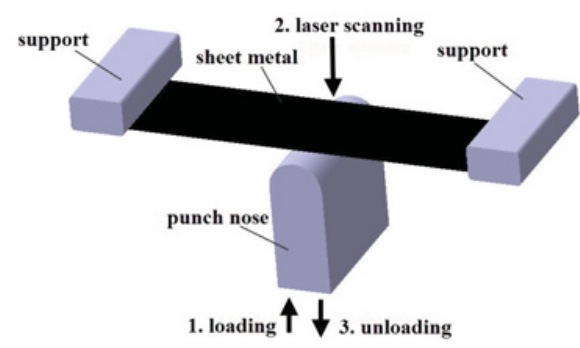

(b)

Figure 1. Two forms of this process: (a) mould bending, (b) three-point bending.

\footnotetext{
${ }^{\text {a }}$ Corresponding author: space@buaa.edu.cn
}

This is an Open Access article distributed under the terms of the Creative Commons Attribution License 4.0, which permits unrestricted use, distribution, and reproduction in any medium, provided the original work is properly cited. 


\section{MATEC Web of Conferences}

In contrast with traditional bending processes, the main advantages of this process are: (1) good forming ability, (2) no significant influence on material structure, (3) good surface quality, (4) good repeatability. This process provides a new way for bending sheet metals. It also provides a solution for forming many sheet metals which are hard to bend by traditional processes because of their complicated structures or special materials, such as integral stiffened panels and titanium alloy sheets.

\section{Research review}

In 1995, Vollertsen et al. [2] presented that small structures could be bended by the buckling mechanism of laser bending. They provided the models of laser bending sheet metals with pre-stresses and some of the experimental results. This is the earliest form of laser bending process of pre-loaded sheet metal. From then on, its concept has been known well gradually and a group of scholars have been attracted for this process. Their researches focused on theoretical researches, forming experiments, numerical simulations and material performance studies of formed sheets.

\subsection{Theoretical researches}

In 2007, Chen et al. [1, 3] proposed the forming mechanism of this process. Pre-bending causes the elastic strain energy to turn up inside the sheet metal, and then the heating effect of laser reduces the yield limit of sheet metal, so that plastic deformation occurs. A portion of elastic strain energy is translated into plastic strain by laser scanning during the deformation. Obviously, this mechanism is different from that of laser bending. Besides, they compared laser bending process of preloaded sheet metal with creep forming and pre-stress peen forming in theory. They found this process has many advantages and concluded that it has great value on the forming of integral stiffened panels.

In 2009, Liu et al. [4] also studied its forming mechanism. They presented that thermal stress which turns up during forming is another factor which affects forming. They thought the integration of thermal stress and pre-stress affect the forming effect, only that pre-stress acts as the main role.

\subsection{Forming experiments and numerical simulations}

According to different types of pre-bending, the forming experiments and numerical simulations are summarized as follows.

\subsubsection{Cantilever sheet bending}

Cantilever sheet bending is the most basic form of bending. Choosing this bending as pre-bending for laser bending process of sheet metal, Guan et al. [5] studied the forming laws with finite element software MSC/MARC in 2002. Their forming model is shown in Fig. 2. Their results showed that the forming process can greatly improve the forming effect. The direction of plastic bending can be controlled if only the direction and degree of pre-bending are controlled reasonably.

To study the effect of different types of pre-stresses on forming, Yao et al. [6] did a research by ANSYS software. Their forming model is similar to model in Fig. 2. The research results revealed that if pre-stress of laser scanning areas is tensile stress, the bending angle of sheet will increase. If pre-stress of laser scanning areas is compressive stress, the bending angle of sheet will decrease.

In order to obtain large bending angle, Roohi et al. [7] carried out an experiment in which a small sheet metal was bended by laser bending process of preloaded sheet metal (Fig. 3). The pre-bending type of sheet metal in the experiment can be regarded as cantilever sheet bending. In the experiment, central region of sheet was fixed by clamps and two sides of the sheet were lifted by punch nose for 


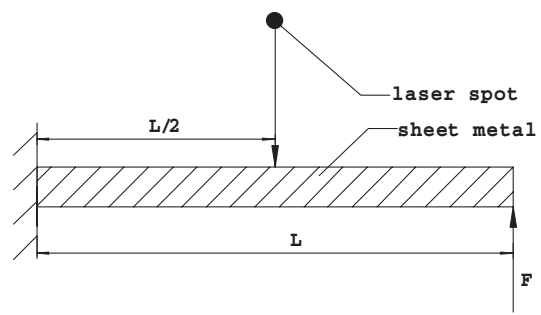

Figure 2. The forming model of Guan.

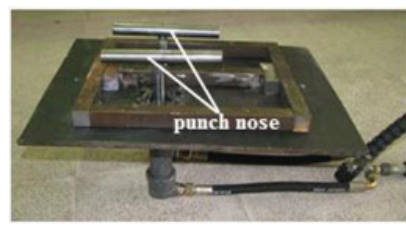

(a)

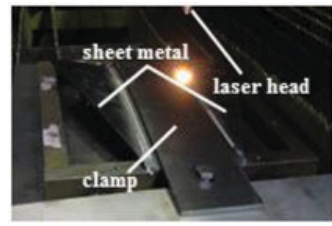

(b)

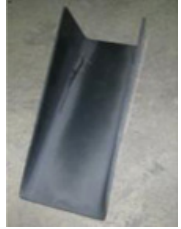

(c)

Figure 3. The experiment of Roohi: (a) pre-bending platform, (b) whole experimental platform, (c) formed sheet.

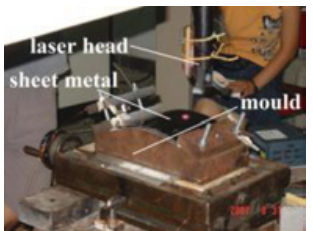

(a)

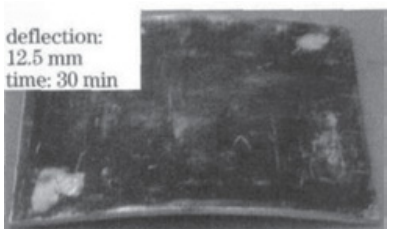

(b)

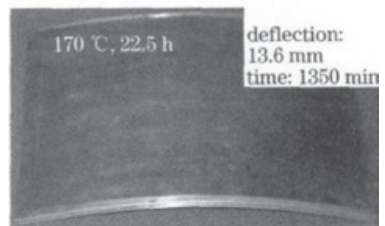

(c)

Figure 4. The experiment of Peng: (a) experimental devices, (b) sample of this process, (c) creep forming sample.

obtaining pre-stress. Then bending areas of the sheet metal were scanned by laser. Finally, 90-degree bending angles were gained on two sides of the sheet metal.

Later, Roohi et al. [7] did a simulation research for achieving a good understanding of the process. Their research showed that the bending angle of sheet increases when the scan pass numbers increase. Change of the equivalent plastic strain inside sheet metal is oscillating in process of forming.

\subsubsection{Mould bending}

In 2009, Peng et al. [8] did an experiment by $\mathrm{CO}_{2}$ laser and gained the result shown in Fig. 4. Comparing with creep forming, laser bending process of preloaded sheet metal spent much less time than creep forming did for gaining similar forming effect.

\subsubsection{Three-point bending}

Peng et al. $[9,10]$ studied a forming model by finite element method. In their model, the sheet was bended by three-point bending and central region of the sheet was scanned by laser. The effects of prestress and laser parameters were investigated. The results revealed that a higher pre-stress lever, or a larger laser spot radius, or a higher laser power, results in a higher forming efficiency. 


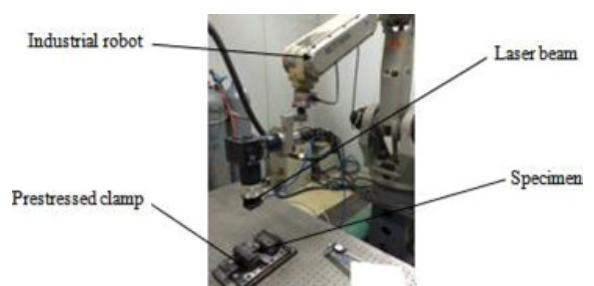

(a)

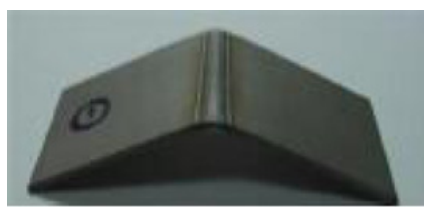

(b)

Figure 5. The experimental platform of Wang: (a) whole experimental platform, (b) formed sheet.

\subsubsection{Horizontal extrusion bending}

Titanium alloy sheets have low plasticity and large spring-back under normal temperature so they are hard to be formed at normal temperature. Laser bending experiment of preloaded titanium alloy sheet was done by Wang et al. shown in Fig. 5 [11]. Experimental results showed that the pre-bending sheet which should recover to its original shape gains an obvious plastic bending after laser scanning and unloading. This proved that laser bending of preloaded sheet metal is effective.

The above experiment process was simulated by ABAQUS software. The result showed that transmission efficiency of the elastic energy could reach to the maximum value through adjusting technological parameters of the laser system without damaging the surface of the sheet. The deformation accuracy of the specimen could also be improved through this approach.

\subsection{Material performance studies of formed sheets}

For engineering parts, their material performance is a key indicator of deciding whether qualified or not. So, the material performance of laser scanning areas must meet performance requirements if we want to take this process for engineering applications [12].

In order to prevent the material performance of sheet metals from being affected too much, the highest temperature of sheets is usually controlled below transformation temperature of their materials. This feature is similar to laser bending. Laser rapid heating effect on materials of laser bending is studied by a group of scholars [13-18]. The research of Shen [16] is typical among these studies. His research results showed that laser scanning could enhance the material strength and reduce the material plasticity. Material fatigue life increases with the increase of laser power, scan pass numbers and the reduction of scanning speed.

Adding the factor of pre-stress, the coupling effect of pre-stress and laser rapid heating is somewhat different from laser heating effect. To study this coupling effect on material performance, Wang et al. [19] studied the material performance of aluminium alloy 7475-T7351 sheets which were bended by this process. Their results showed that the microstructures of formed sheets were not varied obviously, but their micro-hardness and extensive residual stress decreased slightly.

\section{Experimental studies based on flexible pre-bending platform}

It can be seen from the review that the experimental researches of laser bending process of preloaded sheet metal are few. Moreover, the pre-bending platforms that used in these experiments can only work on few simple pre-bending types and small sized sheet metals $[7,8,11]$.

Considering these factors, a large and flexible pre-bending platform has been developed by authors [20], as shown in Fig. 6. It can provide many types of pre-bending, such as multipoint bending, 


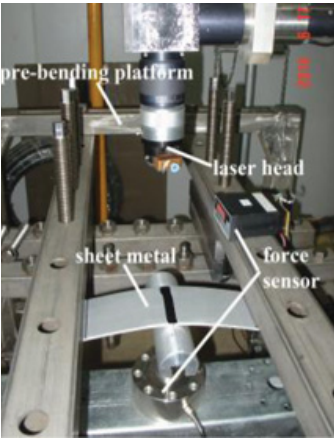

(a)

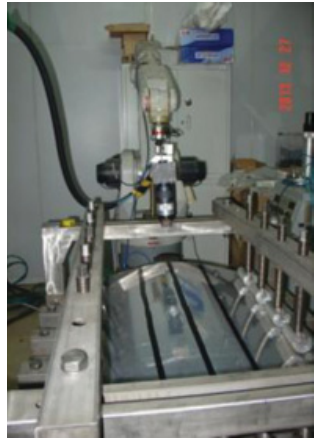

(b)

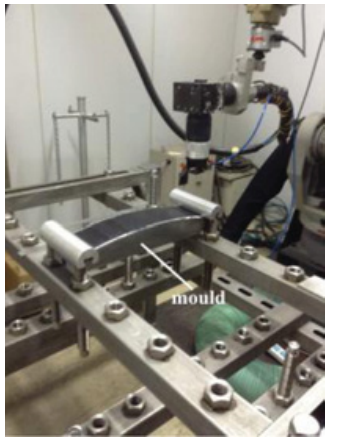

(c)

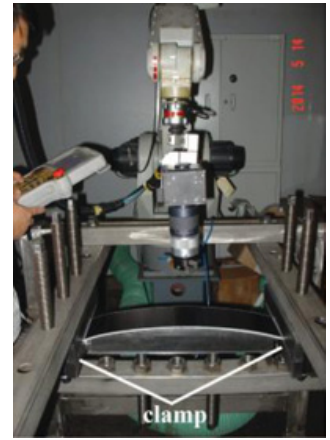

(d)

Figure 6. Different pre-bending types on platform of laser bending process of preloaded sheet metal: (a) three-point bending, (b) multipoint bending, (c) mould bending, (d) stretch bending.

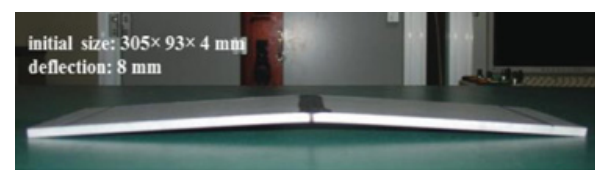

(a)

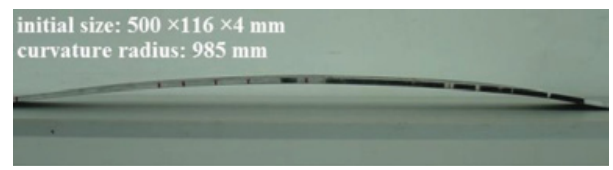

(c)

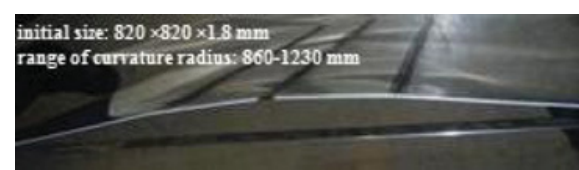

(b)

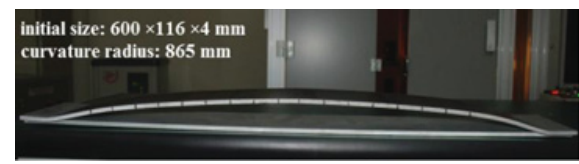

(d)

Figure 7. Corresponding formed sheets of Fig. 6.

mould bending and stretch bending. Moreover, it can bend large sized sheet metals. Furthermore, it can setup force sensors, dial indicators and other measuring devices for experimental data collection.

Many experiments have been done on this platform. Here are the samples.

In Fig. 6a, a sheet was bended by three-point bending and then its central region was scanned by laser. In order to study the changing laws of elastic energy inside the sheet, a force sensor was installed for data collection. Finally, a single curvature sheet was gained, as shown in Fig. 7a.

In Fig. 6b, a large sheet was bended by multipoint bending to gain a variable curvature sheet. The ideal field of pre-stress was obtained and corresponding regions which are black on sheet in Fig. 6b were scanned by laser. Range of curvature radius of the formed sheet is $860-1230 \mathrm{~mm}$, as shown in Fig. $7 \mathrm{~b}$.

In Fig. 6c, a cylindrical mould was fixed on the platform and a sheet was bended by the mould. Uniform pre-stress through thickness direction of sheet was obtained and top surface of sheet was scanned by laser integrally. A cylindrical sheet was gained and its curvature radius is about $985 \mathrm{~mm}$, as shown in Fig. 7c.

In Fig. 6d, two clamps were fixed on the platform in order to obtain larger deformation of sheet. A similar sheet was clamped on its both sides. After that, the mould used in Fig. $6 \mathrm{c}$ was lifted up and the sheet was bended by stretch bending. Then the whole top surface of sheet was scanned by laser. Finally, a cylindrical sheet was also gained and its curvature radius is about $865 \mathrm{~mm}$, as shown in Fig. 7d.

From above, it can be seen that different pre-bending types lead to bend sheet metals into different curved shapes. 


\section{MATEC Web of Conferences}

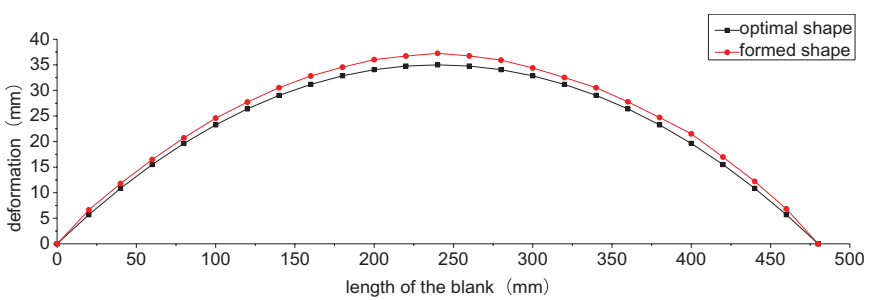

Figure 8. The comparison of the optimal shape and formed shape.

Material of the sheet in Fig. 6b is aluminium alloy 2024-T351 and the formed sheet can be regarded as a scaled model of aircraft skin. The formed sheet has good precision and good smoothness of curvature. Material of the sheet in Fig. 6d is aluminium alloy 2219-T6 and the formed sheet is the scaled model of a part of launch vehicle. Figure 8 shows the comparison of the optimal shape and formed shape. It can be seen that the formed shape is approximate to optimal shape. The forming precision can meet demands of the part. All these show that this process can form large sized structure components of flight vehicles.

\section{Conclusions and further works}

Laser bending process of preloaded sheet metal has great potential value for applications. Many scholars have carried out researches towards this process and they have made some progress. However, these researches are in the initial stage. There is still a long way from engineering application for this forming process. The following researches will be carried out by authors in the future.

(1) Intensive researches of forming theories. Theoretical study helps to reveal the forming laws and provide guidance for applications. It has not been studied out of deducing material constitutive model or analytical model of forming. Besides, the forming mechanism of laser bending process of preloaded sheet metal is not perfect now. All these forming theories will be studied intensively.

(2) Optimization for finite element model. The finite element model of this process involves geometric nonlinear, material nonlinear and contact nonlinear. Moreover, the mesh size of laser scanning areas needs to be very tiny and the iterative calculations of laser heating coupling pre-stress are complicated. All of these make the finite element model a large size and timeconsuming. Therefore, the finite element model needs to be optimized for increase calculative efficiency.

(3) Experimental studies of forming laws. Different pre-bending types lend to different fields of prestress and different fields of pre-stress lend to different forming effect of sheet. The forming laws of different pre-bending types will be studied base on the flexible pre-bending platform.

(4) Manufacturing the actual engineering parts of flight vehicles. Engineering parts usually have complicated shapes and special demands. The laser parameters and the type of pre-bending should be chosen reasonably for forming. Studies will be carried out by finite element method and experiments on the flexible pre-bending platform.

This research is supported by the fundamental Research Funds for the central University and National Natural Science Foundation of China (51005009). The authors would like to acknowledge X.B. Wang, J. Guo and Y. Liang in the Institute of Laser Engineering, Beijing Polytechnic University, China, for their assistance during the experimental stage of this work. 


\section{References}

[1] G.N. Chen, X.F. Wang, G.X. Luo, et al, CN 100552575C (2007) (in Chinese)

[2] F. Vollertsen, I. Komel, R. Kals, Modell. Simul. Mater. Sci. Eng, 3, 107-119 (1995)

[3] G.N. Chen, G.X. Luo, X.F. Wang, et al, CN 100468244C (2007) (in Chinese)

[4] J. Liu, S. Sun, Y.J. Guan, J. Mater. Process. Technol, 209, 1580-1587 (2009)

[5] Y.J. Guan, S. Sun, G.Q. Zhao, et al, J. Mater. Process. Technol, 142, 400-407 (2003)

[6] Z.Q. Yao, H. Shen, Y.J. Shi, et al, Comp Mater Sci, 40, 27-32 (2007)

[7] A.H. Roohi, M. Hoseinpour Gollo, H. Moslemi Naeini, J. Manuf. Processes, 14, 269-276 (2012)

[8] Q. Peng, G.N. Chen, et al, Acta Aeronautica et Astronautica Sinica, 30, 8 (2009) (in Chinese)

[9] Q. Peng, G.N. Chen, X.F. Wang, Chinese Journal of Lasers, 36, 5 (2009) (in Chinese)

[10] Q. Peng, G.N. Chen, M.X. Wang, et al, Chinese Journal of Lasers, 38, 10 (2011) (in Chinese)

[11] X.F. Wang, D.X. Liu, W.D. Li, et al, Manuf. Rev, 1, 25 (2014)

[12] R.S. Piascik, S.A. Willard, Fatigue Fract. Eng. Mater. Struct, 17, 11 (1994)

[13] M. Merklein, T. Hennige, M. Geiger, J. Mater. Process. Technol, 115, 159-165 (2001)

[14] G. Thomson, M. Pridham, J. Mater. Process. Technol, 118, 40-44 (2001)

[15] L.Q. Li, Y.B. Chen, L.W. Zhang, et al., Chin J Nonferrous Met, 15, 6 (2005) (in Chinese)

[16] H. Shen, Study on Shape Forming in Laser Forming, PhD dissertation, Shanghai Jiao Tong University (2007) (in Chinese)

[17] X.Q. Zhang, Y.K. Zhang, J.Z. Zhou, et al, Chinese Journal of Lasers, 35, 7 (2008) (in Chinese)

[18] K. Maji, D.K. Pratihar, A.K. Nath, Opt. Laser Technol, 49, 18-27 (2013)

[19] M.X. Wang, G.N. Chen, Q. Peng, Acta Aeronautica et Astronautica Sinica, 32, 7 (2011) (in Chinese)

[20] W.D. Li, Z.L. Wen, L. Zhang, et al, CN 103551469A (2014) (in Chinese) 